\title{
ON TREE-LIKE CONTINUA WHICH ARE HOMOGENEOUS WITH RESPECT TO CONFLUENT LIGHT MAPPINGS
}

\author{
PAWEL KRUPSKI \\ (Communicated by James E. West)
}

\begin{abstract}
If $X$ is a tree-like continuum with property $K$ which is homogeneous with respect to confluent light mappings, then $X$ contains no two nondegenerate subcontinua with the one-point intersection. This is a generalization of C. L. Hagopian's result concerning homogeneous $X$.
\end{abstract}

\section{INTRODUCTION}

A space $X$ is homogeneous with respect to a class $M$ of mappings if for every two points $x, y \in X$ there exists a mapping $f \in M$ of $X$ onto $X$ such that $f(x)=y$.

Many results concerning the generalized homogeneity have been obtained in recent years and a special interest was given to generalize theroems on homogeneous continua for some (larger than homeomorphisms) classes $M$ of mappings (see [2]).

There are known one-dimensional continua which are homogeneous with respect to open light mappings but are not homogeneous (e.g. the one-point union of two Menger universal curves [2, p. 588]). However, we don't know such an example of a tree-like continuum. It was observed in [5] that if a continuum $X$ is homogeneous with respect to open mappings and each proper subcontinuum of $X$ is an arc, then $X$ is not tree-like. Moreover, J. R. Prajs has recently proved that $X$ is a solenoid, [8].

In this paper we present an immediate proof that no tree-like continuum with the property of Kelley which is homogeneous with respect to confluent light mappings contains two nondegenerate subcontinua with the one-point intersection. In particular, a tree-like continuum which is homogeneous with respect to open light mappings contains no two nondegenerate subcontinua with the onepoint intersection. The theorem is a generalization of Hagopian's result about

Received by the editors July 15, 1988.

1980 Mathematics Subject Classification (1985 Revision). Primary 54F20; Secondary 54F50, $54 \mathrm{C} 10$.

Key words and phrases. Continuum, homogeneous, tree-like, property $K$, confluent mapping, Effros' theorem, outlet point. 
homogeneous $X$ (see [3 and 6]). On the other hand, the theorem with some ideas of its proof have been used to prove that homogeneous tree-like continua are hereditarily indecomposable (see [7]).

\section{Preliminaries}

Throughout the paper all spaces are metric and all mappings are continuous. $C(X)$ denotes the hyperspace of all nonvoid subcontinua of a compact space $X$ with the Hausdorff distance.

Let us recall that a mapping $f$ of a compact space $X$ onto $Y$ is confluent if for every $A \in C(Y)$ and for every component $B$ of $f^{-1}(A)$ we have $f(B)=A$. It is known that open mappings between compact spaces are confluent.

If a point $a \in X$ is fixed, we define the evaluation mapping $T_{a}: X^{X} \rightarrow X$ by $T_{a}(f)=f(a)$. Recall that the quasi-interior of $A \subset X$ is the set $A^{*}=\bigcup\{U: U$ is open in $X$ and $U-A$ is of the first category $\}$. The following theorem holds [2, p. 584].

Proposition 2.1. If a compact space $X$ is homogeneous with respect to a Borel set $M \subset X^{X}$ and $a \in X$, then there is an $h \in M$ such that $h(X)=X$ and $h(a) \in T_{a}(H)^{*}$ for each $H$ open in $M$ with $h \in H$.

A continuum $A$ of a proper subcontinuum $B$ of $X$ is called an outlet subcontinuum of $B$ if $A \subset Z$ for every subcontinuum $Z$ of $X$ such that $Z \cap B \neq \varnothing \neq Z-B$. If $A=\{a\}$, then $a$ is called an outlet point of $B$ [6].

The following propositions follow easily from the definitions.

Proposition 2.2. If $f$ is a confluent mapping of $X$ and $A$ is an outlet subcontinuum of $B \in C(X)$, then $f(A)$ is an outlet subcontinuum of $f(B)$.

Proposition 2.3. Suppose $Y$ is a subcontinuum of a hereditarily unicoherent continuum $X$ and $Y=K \cup L$, where $K$ and $L$ are subcontinua of $X$ with distinct outlet point $p$ and $q$, respectively, $p \in L$ and $\operatorname{diam} L<\operatorname{diam} K$. Then the subcontinuum of $X$ which is irreducible between $p$ and $q$ is an outlet subcontinuum of $Y$.

Let us recall that a continuum $X$ has the property of Kelley (property $K$ ) if for every $y \in X$, for every sequence $\left\{y_{n}\right\} \subset X$ converging to $y$ and for every $Y \in C(X)$ containing $y$ there exists a sequence $\left\{Y_{n}\right\} \subset C(X)$ converging to $Y$ such that $y_{n} \in Y_{n}, n=1,2, \ldots$. Continua which are homogeneous with respect to open mappings have property $K[1$, p. 380] but homogeneity with respect to confluent mappings does not imply property $K$ [4].

Proposition 2.4. If a continuum $X$ has property $K$ and $A_{k}$ is an outlet subcontinuum of $B_{k} \in C(X), k=1,2, \ldots, \operatorname{Lim}_{k} A_{k}=A, \operatorname{Lim}_{k} B_{k}=B$, then $A$ is an outlet subcontinuum of $B$.

A finite collection $\mathscr{C}$ of open subsets of $X$ is called a tree-chain if its nerve is a tree. Elements of $\mathscr{C}$ are called links. A link $C \in \mathscr{C}$ is an end-link if 
$\mathscr{C}-\{C\}$ is again a tree-chain; if $C$ is not an end-link of $\mathscr{C}$, then $C$ separates $\mathscr{C}$ and maximal tree-chains in $\mathscr{C}-\{C\}$ are called branches of $\mathscr{C}-\{C\}$. A continuum $X$ is tree-like if there exists a sequence of tree-chains $\mathscr{C}_{n}, n=$ $1,2, \ldots$, covering $X$ such that $\mathscr{C}_{n+1}$ refines $\mathscr{C}_{n}$ and $\lim _{n}$ mesh $\mathscr{C}_{n}=0$. The sequence $\left\{\mathscr{C}_{n}\right\}$ will be called a defining sequence of $X$. By the diameter of a tree-chain $\mathscr{C}$ we understand the number $\operatorname{diam} \bigcup \mathscr{C}$.

\section{OUTLET POINTS IN TREE-LIKE CONTINUA}

Lemma 3.1. Let $\alpha$ be a positive number and let $\mathscr{D}$ be a tree-chain in a space $X$ such that mesh $\mathscr{D}<\alpha / 3, \alpha<\operatorname{diam} \bigcup \mathscr{D}$. Denote by $\mathscr{D}^{\circ}$ the family of all branches of $\mathscr{D}-\{C\}$ having diameters $<\alpha / 3$, where $C$ runs separating links of $\mathscr{D}$. Let $\mathscr{D}^{\prime}$ be the set of all such $C \in \mathscr{D}$ that there exists a branch of $\mathscr{D}-\{C\}$ (called associated with $C$ ) which is a maximal element of $\mathscr{D}^{\circ}$ with respect to inclusion. Fix an end-link $D \in \mathscr{D}$. Then there is $C \in \mathscr{D}^{\prime}$ such that an associated branch of $\mathscr{D}-\{C\}$ does not contain $D$ and exactly one branch $\mathscr{B}$ of $\mathscr{D}-\{C\}$ has the diameter $\geq \alpha / 3 ;$ moreover, $D \in \mathscr{B}$.

Proof. Suppose the contrary. Take $C_{1} \in \mathscr{D}^{\prime}$, an associated branch of which does not contain $D$ (clearly, it exists) and let $\mathscr{B}_{1}$ be a branch of $\mathscr{D}-\left\{C_{1}\right\}$ of diameter $\geq \alpha / 3$ such that $D \notin \mathscr{B}_{1}$. Again, choose $C_{2} \in \mathscr{D}^{\prime} \cap \mathscr{B}_{1}$, an associated branch of which is in $\mathscr{B}_{1}$ (so, it does not contain $D$ ) and let $\mathscr{B}_{2}$ be a branch of $\mathscr{D}-\left\{C_{2}\right\}$ of diameter $\geq \alpha / 3$ such that $D \notin \mathscr{B}_{2}$, etc. Continuing this process we get an infinite set $\left\{C_{1}, C_{2}, \ldots\right\}$, a contradiction.

In the remaining part of this section, $X$ will denote a nondegenerate tree-like continuum with defining sequence $\left\{\mathscr{C}_{n}\right\}$.

A subcontinuum $Y$ of $X$ is said to be a limit of branches if there are branches $\mathscr{D}_{n_{k}}$ of $\mathscr{C}_{n_{k}}-\left\{E_{k}\right\}$ for some separating links $E_{k} \in \mathscr{C}_{n_{k}}, n_{1}<n_{2}<\cdots$, such that $Y=\operatorname{Lim}_{k} \cup \mathscr{D}_{n_{k}}$.

Lemma 3.2. There is a sequence $\left\{Y_{k}\right\}, k=1,2, \ldots$, of nondegenerate subcontinua of $X$ such that for each $k$

(i) $Y_{k}$ is a limit of branches,

(ii) $Y_{k+1} \subset Y_{k}$,

(iii) $\bigcap_{k=1}^{\infty} Y_{k}$ is degenerate.

Proof. Clearly, $X$ contains a nondegenerate limit of branches $Y_{1}$. Suppose nondegenerate subcontinua $Y_{1}, Y_{2}, \ldots, Y_{m-1}=Y$ of $X$ satisfy (i) and (ii), $0<\alpha<\operatorname{diam} Y / 2$. To prove the lemma we shall construct a nondegenerate limit of branches $Y_{m} \subset Y$ with $\alpha / 3 \leq \operatorname{diam} Y_{m} \leq \alpha$. Without loss of generality we can assume $Y=\operatorname{Lim}_{n} \cup \mathscr{D}_{n}$, where $\mathscr{D}_{n}$ is a branch of $\mathscr{C}_{n}-\left\{E_{n}\right\}$ for a separating link $E_{n} \in \mathscr{C}_{n}$ and mesh $\mathscr{D}_{n}<\alpha / 3<\alpha<\operatorname{diam} \cup \mathscr{D}_{n}$. Let $D_{n}$ be an end-link of $\mathscr{D}_{n}$ disjoint with $E_{n}$. By Lemma 3.1, for each $n$ there exists $C_{n} \in \mathscr{D}_{n}^{\prime}$ such that an associated branch of $\mathscr{D}_{n}-\left\{C_{n}\right\}$ does not contain $D_{n}$ and exactly one branch $\mathscr{B}_{n}$ of $\mathscr{D}_{n}-\left\{C_{n}\right\}$ has the diameter $\geq \alpha / 3$; moreover, $D_{n} \in \mathscr{B}_{n}$. Then a link $B_{n} \in \mathscr{B}_{n}$ intersects $C_{n}$ and the branch $\mathscr{A}_{n}$ of $\mathscr{D}_{n}-\left\{B_{n}\right\}$ 
containing all branches of $\mathscr{D}_{n}-\left\{C_{n}\right\}$ which are different from $\mathscr{B}_{n}$ is also a branch of $\mathscr{C}_{n}-\left\{B_{n}\right\}$ satisfying $\alpha / 3<\operatorname{diam} \cup \mathscr{A}_{n}<\alpha$. Finally, put $Y_{m}=$ $\operatorname{Lim}_{k} \bigcup \mathscr{A}_{n_{k}}$ for a convergent subsequence $\operatorname{cl}\left(\bigcup \mathscr{A}_{n_{k}}\right), k=1,2, \ldots$.

Lemma 3.3 (See [6, Lemma 3.2]). If $X$ has property $K$ and $Y \in C(X)$ is a limit of branches, then $Y$ contains an outlet point.

Lemmas 3.2 and 3.3 imply a stronger version of [6, Theorem 3.3].

Theorem 3.4. If $X$ has property $K$, then $X$ contains a sequence of nondegenerate subcontinua $Y_{k}$ such that for each $k=1,2, \ldots$

(i) $Y_{k}$ has an outlet point,

(ii) $Y_{k+1} \subset Y_{k}$,

(iii) $\bigcap_{k=1}^{\infty} Y_{k}$ is degenerate.

\section{Main Results}

Theorem 4.1. If a nondegenerate tree-like continuum $X$ with property $K$ is homogeneous with respect to confluent light mappings, then there exists a sequence $Z_{k} \in C(X)$ such that, for each $k, Z_{k}$ contains a nondegenerate outlet subcontinuum, $\operatorname{diam} Z_{k}<1 / k$ and $\bigcap_{k=1}^{\infty} Z_{k} \neq \varnothing$.

Proof. Theorem 3.4 guarantees the existence of a sequence $\left\{Y_{k}\right\}$ satisfying (i)(iii). It suffices to consider the following two cases: (1) the only point $p$ of $\bigcap_{k=1}^{\infty} Y_{k}$ is an outlet point of $Y_{k}$ for each $k$; (2) for every such a sequence $\left\{Y_{k}\right\}$, each $Y_{k}$ contains an outlet point $p_{k} \notin \bigcap_{k=1}^{\infty} Y_{k}$.

In case (1) let $h$ be a confluent light mapping of $X$ onto $X$ which is described in Proposition 2.1 for the class $M$ of all confluent light mappings and for $a=p$. Each $h\left(Y_{k}\right)$ is nondegenerate. By Proposition 2.2, $h(p)$ is an outlet point of $h\left(Y_{k}\right)$ for each $k$. Furthermore, $\{h(p)\}=\bigcap_{k=1}^{\infty} h\left(Y_{k}\right)$ and $h\left(Y_{k+1}\right) \subset$ $h\left(Y_{k}\right)$. Fix a positive integer $k$. Let $m$ be such that $\operatorname{diam} h\left(Y_{m}\right)<1 / 4 k$ and let $0<\varepsilon<\operatorname{diam} h\left(Y_{m}\right) / 2$. There exists a $\delta>0$ such that $B(h(p), \delta) \subset$ $T_{p}(H)^{*}$, where $B(h(p), \delta)$ denotes the $\delta$-ball about $h(p)$ and $H$ is the $\varepsilon$ ball about $h$ in $M$. Let $n$ be such that $n>m, h\left(Y_{n}\right) \subset B(h(p), \delta)$ and $\operatorname{diam} h\left(Y_{n}\right)<\operatorname{diam} h\left(Y_{m}\right)-2 \varepsilon$. Choose $q \in h\left(Y_{n}\right)-\{h(p)\}$. Since $q \in T_{p}(H)^{*}$ and $T_{p}(H)$ is dense in $T_{p}(H)^{*}$, there exists a sequence of points $q_{s} \in T_{p}(H)$, $s=1,2, \ldots$, converging to $q$. Hence, for each $s$ there is $h_{s} \in H$ sending $p$ onto $q_{s}$. Take a convergent subsequence $h_{s_{i}}\left(Y_{m}\right), i=1,2, \ldots$, and denote its limit by $K$. In view of Proposition 2.2, $q_{s_{i}}$ is an outlet point of $h_{s_{i}}\left(Y_{m}\right)$ and, by Proposition 2.4, $q$ is an outlet point of $K$. Note that $\operatorname{diam} h\left(Y_{n}\right)<\operatorname{diam} K$. Define $Z_{k}=h\left(Y_{n}\right) \cup K$. Observe that $\operatorname{diam} Z_{k} \leq 2 \operatorname{diam} h\left(Y_{m}\right)<1 / 2 k$. We conclude by Proposition 2.3 that the unique subcontinuum of $X$ irreducible between $p$ and $q$ is a nondegenerate outlet subcontinuum of $Z_{k}$. The above construction of $Z_{k}$ shows that $h(p) \in Z_{k}$ for each $k$.

Assume now case (2) holds. Let $Y_{k} \in C(X)$ form a decreasing sequence with outlet points $p_{k} \in Y_{k}, \bigcap_{k=1}^{\infty} Y_{k}=\{p\}, p_{k} \neq p$ for each $k$. Fix a positive 
integer $k$. Take $Y_{n}$ with $\operatorname{diam} Y_{n}<1 / 2 k$. There exists a confluent light mapping $f$ of $X$ onto $X$ such that $f(p)=p_{n}$. Subcontinua $f\left(Y_{m}\right), m=$ $1,2, \ldots$, form again a sequence satisfying Theroem 3.4 and $\bigcap_{m=1}^{\infty} f\left(Y_{m}\right)=$ $\left\{p_{n}\right\}$. Let $m$ be such that $\operatorname{diam} f\left(Y_{m}\right)<\operatorname{diam} Y_{n}$. Assuming (2), $f\left(Y_{m}\right)$ contains an outlet point $q_{m}$ different from $p_{n}$. In view of Proposition 2.3, the continuum $Z_{k}=Y_{n} \cup f\left(Y_{m}\right)$ contains a nondegenerate outlet subcontinuum. We have $\operatorname{diam} Z_{k}<1 / k$ and $p \in Z_{k}$ for each $k$.

So, the proof is complete.

The following theorem is an easy consequence of Theorem 4.1 and Proposition 2.2.

Theorem 4.2. No tree-like continuum with property $K$ which is homogeneous with respect to confluent light mappings contains two nondegenerate subcontinua with the one-point intersection.

Corollary 4.3. No tree-like continuum which is homogeneous with respect to open light mappings contains two nondegenerate subcontinua with the one-point intersection.

Recall that $A \in C(X)$ is weakly terminal if there is a $\delta>0$ such that if $Z \in C(X), \operatorname{diam} Z<\delta$ and $Z \cap A \neq \varnothing$, then $Z \subset A$ [6]. It is easy to see (compare [6, Lemma 4.1]) that the continua $Z_{k}$ in Theorem 4.1 are weakly terminal. So, we have the following generalization of [6, Theorem 4.2].

Corollary 4.4. Each nondegenerate tree-like continuum with property $K$ which is homogeneous with respect to confluent light mappings contains arbitrarily small, weakly terminal subcontinua with nondegenerate outlet subcontinua.

If $A \subset X$ is an outlet subcontinuum of itself, then $A$ is called a terminal subcontinuum of $X$.

Question 4.5. Suppose $X$ is a tree-like nondegenerate continuum with property $K$ which is homogeneous with respect to confluent light mappings. Does $X$ contain arbitrarily small, nondegenerate, terminal (indecomposable) subcontinua? Is $X$ indecomposable?

\section{REFERENCES}

1. J. J. Charatonik, The property of Kelley and confluent mappings, Bull. Polish. Acad. Sci. Math. 31 (1983), 375-380.

2. J. J. Charatonik and T. Maćkowiak, Around Effros' theorem, Trans. Amer. Math. Soc. 298 (1986), pp. 579-602.

3. C. L. Hagopian, No homogeneous tree-like continuum contains an arc, Proc. Amer. Math. Soc. 88 (1983), pp. 560-564.

4. H. Kato, Generalized homogeneity of continua and a question of J. J. Charatonik, Houston J. Math. 13 (1987), 51-63.

5. P. Krupski On continua each of whose proper subcontinua is an arc-a generalization of two theroems of R. H. Bing, Colloq. Math. Soc. János Bolyai 41, Topology and Applications, Eger (Hungary), 1983, pp. 367-371. 


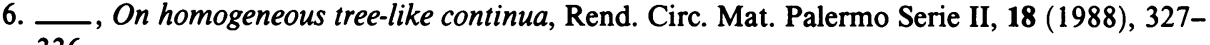
336.

7. P. Krupski and J. R. Prajs, Outlet points and homogeneous continua, Trans. Amer. Math. Soc. (to appear).

8. J. R. Prajs, Openly homogeneous continua having only arcs for proper subcontinua, preprint.

Mathematical Institute, University of Wroclaw, Pl. Grunwaldzki $2 / 4,50-384$ Wroclaw, Poland 